\title{
On ring theoretic lattice modules
}

\author{
by \\ David G. Whitman (Riverside, Calif.)
}

The lattice of ideals of a commutative Noetherian ring with identity has been abstracted by the notion of a Noether lattice. This note generalizes this by presenting an abstraction of the lattice of submodules of a unitary module over a commutative ring with identity. The basic idea is to extend R. P. Dilworth's formulation of a principal. element in a multiplicative lattice into a module setting.

Section 1 gives basic definitions and some examples. Section 2 presents an abstract version of Nagata's principle of idealization thereby enabling much of the theory of Noether lattices to be extended immediately to Noetherian ring theoretic modules. In Section 3 form modules and localization are investigated.

§ 1. Ring theoretic lattice modules. Let $L$ be a complete modular lattice with maximum element $I$ and minimum element $O$ and with a multiplication satisfying:

$$
\begin{gathered}
(A B) C=A(B C), \\
A B=B A, \\
\left(\bigvee_{\alpha} A_{a}\right)\left(\bigvee_{\beta} B_{\beta}\right)=\bigvee_{a, \beta} A_{a} B_{\beta}, \\
I A=A .
\end{gathered}
$$

Under these conditions $L$ is said to be multiplicative. An $L$-module $M$ is a complete modular lattice with maximum element $\mathrm{mt}$ and minimum element $O_{M}$, and which admits a (left) multiplication from $L$ satisfying:

$$
\begin{gathered}
(A B) N=A(B N), \\
\left(\bigvee_{a} A_{\alpha}\right)\left(\bigvee_{\beta} N_{\beta}\right)=\bigvee_{a, \beta} A_{\alpha} N_{\beta}, \\
I N=N \quad \text { and } \quad O N=O_{M} .
\end{gathered}
$$

Associated with an $L$-module $M$ are operations called residual division. If $A \in L$ and $N \in M$, then $N: A$ is the join of all $N^{\prime} \in M$ such 
that $A N^{\prime} \leqslant N$. Similarly if $N$ and $N^{\prime}$ belong to $M$, then $N: N^{\prime}$ is the join of all $A \in L$ such that $A N^{\prime} \leqslant N$.

Now in order to abstract the notion of a module over a commutative ring it is necessary to formulate an abstraction of a principal element. The following definition is due to E. W. Johnson [3]. An element $N$ of an $L$-module $M$ is said to be principal if for arbitrary $A \in I$ and $N^{\prime} \in M$ the following holds:

$$
\begin{gathered}
\left(A \wedge\left(N^{\prime}: N\right)\right) N=A N \wedge N^{\prime}, \\
\left(N^{\prime} \vee A N\right): N=\left(N^{\prime}: N\right) \vee A .
\end{gathered}
$$

An $L$-module $M$ is said to be principally generated if every element is a join of principal elements. A principally generated multiplicative lattice satisfying the ascending chain condition is called a Noether lattice.

For the remainder of this paper $L$ will denote a principally generated multiplicative lattice. A principally generated $L$-module is said to be ring theoretic provided every element of $L$ is a join of principal elements $A$ which satisfy the following for all $N_{1}, N_{2} \in M$ :

$$
\begin{aligned}
& \left(N_{1} \vee A N_{2}\right): A=N_{1}: A \vee N_{2} \\
& A\left(N_{1} \wedge N_{2}: A\right)=A N_{1} \wedge N_{2}
\end{aligned}
$$

Such elements of $L$ will be called $M$-principal. A ring theoretic module is said to be Noetherian if it satisfies the ascending chain condition. For the remainder of this paper, $M$ will denote a ring theoretic $L$-module. Arbitrary elements of $L$ will be denoted $A, B, C, \ldots$ and principal elements $a, b, c, \ldots$ Arbitrary elements of $M$ will be denoted $N, N_{1}, N_{2}, \ldots$ and principal elements $n, n_{1}, n_{2}, \ldots$

Examples of principally generated multiplicative lattices and ring theoretic lattice modules are abundant. The lattice $L(R)$ of graded ideals of a graded commutative ring $R$ with identity is a principally generated multiplicative lattice, and the set of graded $R$-submodules of a graded unitary $R$-module is a ring theoretic $L(R)$-module. Furthermore, an arbitrary (possibly non-commutative) ring with identity which satisfies the ascending chain condition on ideals has a principally generated multiplicative lattice for an ideal system provided:

(1.12) $A B=B A$, for ideals $A, B$ of $R$,

(1.13) Every ideal is a join of principal ideals $R a R$ such that $R a R$ $=a R=R a$.

Verification of the above statements is straightforward.

The following lemma cites some elementary facts about $M$.
LEMMA 1.1. The following hold for $L$ and $M$ :

(1) $\bigwedge_{a}\left(N_{a}: N\right)=\left(\bigwedge_{a} N_{a}\right): N, \bigwedge_{a}\left(N_{a}: A\right)=\left(\bigwedge_{a} N_{a}\right): A$.

(2) $N: \bigvee_{a} N_{a}=\bigwedge_{a}\left(N: N_{a}\right), N: \bigvee_{a} A_{a}=\bigwedge_{a}\left(N: A_{a}\right)$

(3) $N: A B=(N: A): B, N: A N_{1}=(N: A): N_{1}=\left(N: N_{1}\right): A$.

(4) If $A \leqslant B$, then $A N \leqslant B N$ and $A N \leqslant N$; and if $N_{1} \leqslant N_{2}$, then $A N_{1} \leqslant A N_{2}$.

(5) $N \geqslant A(N: A), N \geqslant\left(N: N_{1}\right) N_{1}$, and $N: A \geqslant N$.

(6) $I=N_{1}: N_{2}$ if and only if $N_{2} \leqslant N_{1}$.

(7) If $A \leqslant B$, then $N: B \leqslant N: A$; if $N_{1} \leqslant N_{2}$, then $N_{1}: A \leqslant N_{2}: A$ and $N: N_{1} \geqslant N: N_{2}$.

(8) $N_{1}: N_{2}=N_{1}:\left(N_{1} \vee N_{2}\right), N_{1}: N_{2}=\left(N_{1} \wedge N_{2}\right): N_{2}$.

(9) $(A: B) N \leqslant A N: B, A: B \leqslant A N: B N$.

(10) If $n \in M$ is principal, then $n \wedge N=(N: n) n$ and $A n: n=A \vee O_{M}: n$.

(11) If $a \in L$ is $M$-principal and $n \in M$ is principal, then an is principal in $M$.

Proof. The proofs of (1)-(9) are quite simple and may be omitted. (10) may be proved as follows: $n \wedge N=\operatorname{In} \wedge N=(I \wedge N: n) n=(N: n) n$, since $n$ is principal. Also since $n$ is principal, $A n: n=\left(O_{M} \vee A n\right): n$ $=O_{M}: n \vee A$. (11) may be proved in a way very similar to the proof of Corollary 3.3 in [2], q.e.d.

The following theorem generalizes the notions of factor module and submodule.

THEOREM 1. Let $N_{1} / N_{2}$ be an interval of $M$, let $A \leqslant N_{2}: N_{1}$, and let $L / A$ denote the set of $B \in L$ such that $B \geqslant A$. Then $L / A$ is a principally generated multiplicative lattice under the multiplication $B \cdot C=B C \vee A$ and $N_{1} / N_{2}$ is a ring theoretic module over $L / A$ when multiplication is defined by $B \cdot N$ $=B N \vee N_{2}$, for $B \in L / A$ and $N \in N_{1} / N_{2}$.

Proof. It can be easily verified that $L / A$ is a multiplicative lattice. In $[1$, p. 488] Dilworth proved that, when $L$ is Noetherian, if $a$ is principal in $L$, then $a \vee A$ is principal in $L / A$. As his proof does not rely upon the ascending chain condition, it also suffices for the general case. This clearly implies that $L / A$ is principally generated.

It is easily verified that $N_{1} / N_{2}$ is complete, modular, and satisfies (1.5)-(1.7). Every element of $L / A$ is a join of $N_{1} / N_{2}$-principal elements because elements of the form $a \vee A$ where $a$ is $M$-principal are also $N_{1} / N_{2}$ principal in $L / A$. (1.11) is shown as follows: Let $N, N^{\prime} \in N_{1} / N_{2}$. Now $((a \vee A) \cdot N) \wedge N^{\prime}=\left((a \vee A) N \vee N_{2}\right) \wedge N^{\prime}=\left(a N \vee N_{2}\right) \wedge N^{\prime}$ since $A N \leqslant N_{2}$. By 
modularity $\left(a N \vee N_{2}\right) \wedge N^{\prime}=\left(a N \vee N^{\prime}\right) \wedge N_{2}$. Since $a$ is $M$-principal $\left(a N \wedge N^{\prime}\right) \vee N_{2}=a\left(N \wedge N^{\prime}: a\right) \vee N_{2}=(a \vee A) \cdot\left(N \wedge\left(N^{\prime}: a\right)\right)$. Letting $:^{\prime}$ denote residuation with respect to $L / A$ and $N_{1} / N_{2}$, it is easy to compute that $N^{\prime}:^{\prime}(a \vee A)=N^{\prime}:(a \vee A) \wedge N_{1}=\left(N^{\prime}: a\right) \wedge N_{1}$. Therefore $(a \vee A) \cdot N \wedge N^{\prime}$ $=(a \vee A) \cdot N \wedge\left(N^{\prime}:^{\prime}(a \vee A)\right)$ and (1.11) is satisfied. (1.10) can be verified similarly for $a \vee A$. Also it can be shown similarly that, if $n \leqslant N_{1}$, then $n \vee N_{2}$ is principal in $N_{1} / N_{2}$ with respect to $L / A$, q.e.d.

At the end of this section it seems appropriate to pose the following problem: Classify the non-commutative rings which have a Noether lattice for an ideal system.

§ 2. The principle of idealization. Again in this section $L$ will denote a principally generated multiplicative lattice and $M$ a ring theoretic $L$-module. The essential result in this section is the following theorem which is called the principle of idealization after its ring theory counterpart (see $[4$, p. 2]).

THEOREM 2. Let $L \oplus M$ denote the set of all ordered pairs $(A, N)$ where $A \in L$ and $N \in M$ are such that $A \mathfrak{m} \leqslant N$. Under the operations below, $L \oplus M$ is a principally generated multiplicative lattice. Furthermore if $L$ and $M$ are Noetherian, then $L \oplus M$ is a Noether lattice.

$$
\begin{aligned}
\left(A_{1}, N_{1}\right) \vee\left(A_{2}, N_{2}\right) & =\left(A_{1} \vee A_{2}, N_{1} \vee N_{2}\right), \\
\left(A_{1}, N_{1}\right) \wedge\left(A_{2}, N_{2}\right) & =\left(A_{1} \wedge A_{2}, N_{1} \wedge N_{2}\right), \\
\left(A_{1}, N_{1}\right)\left(A_{2}, N_{2}\right) & =\left(A_{1} A_{2}, A_{1} N_{2} \vee A_{2} N_{1}\right) .
\end{aligned}
$$

Proof. The details of proving that $L \oplus M$ is a multiplicative lattice are quite straightforward and will be omitted. It remains to show that $L \oplus M$ is principally generated. To show this it suffices to show that elements of the form $(a, a \mathrm{mt})$ and $(O, n)$ are principal, where a is $M$-principal, since every element of $L \oplus M$ is clearly a join of such elements. The following calculation shows that $(a, a$ m) satisfies $(1.8)$. Let $(A, N)$ and $\left(B, N^{\prime}\right)$ be arbitrary elements of $L \oplus M$. Now it is easy to compute that $\left(B, N^{\prime}\right):(a, a \mathrm{~m})=\left(B: a, N^{\prime}: a\right)$. Hence $\left((A, N) \wedge\left(\left(B, N^{\prime}\right):(a, a \mathrm{~m})\right)\right)(a, a \mathrm{~m})$ $=\left((A, N) \wedge\left(B: a, N^{\prime}: a\right)\right)(a, a \mathfrak{m})$. By definition of meet and product in $L \oplus M$ the latter reduces to $\left((A \wedge B: a) a, a\left(N \wedge N^{\prime}: a\right)\right)$. Since $a$ is $M$-principal, this equals $\left(a A \wedge B, a N \wedge N^{\prime}\right)$. Now $((a, a \mathrm{~m})(A, N)) \wedge\left(B, N^{\prime}\right)$

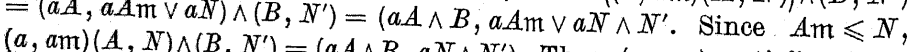
The other identities for $(0, n)$ and $\left.a N \wedge N^{\prime}\right)$. Thus (a, ant) satisfies (1.8). If $L$ and $M$ are $(O, n)$ and $(a, a \mathrm{mt})$ can be verified similarly. will terminate when Noetherian, then any ascending chain $\left\{\left(A_{i} ; N_{i}\right)\right\}$ is a Noether lattice, q.e.d.

The principle of idealization provides a useful technique in developing the theory of Noetherian ring theoretic $L$-modules, where $L$ is a Noether - lattice. For the remainder of this paper $L$ and $M$ will be assumed Noetherian. The following corollaries indicate the usefulness of Theorem 2. A Noether lattice is said to be local if it has a unique maximal prime element.

CoRolLARY 1. Let $L$ be local with unique maximal prime element $P$, and let $N \neq O \in M$. Then every minimal set of principal elements generating $N$ has the same cardinality which is equal to $l(N / P N)$.

Proof. This result is known already in the case $M=L$ (see [2]). Now $(O, N) \in L \oplus M$ which is local with maximal prime element $(P, \mathrm{~m})$. Let $n_{1}, \ldots, n_{k}$ be a minimal set of principal generators for $N$. Hence $(O, N)$ $=\left(O, n_{1}\right) \vee \ldots \vee\left(O, n_{k}\right)$ where each $\left(O, n_{i}\right)$ is principal in $L \oplus M$. So by the already known result about Noether lattices, $k=l((O, N) /(P, \mathrm{~m})(O, N))$ $=l((O, N) /(O, P N))=l(N / P N)$, q.e.d.

CoROLLaRy 2. Let $A \in L$, and let $N, N^{\prime} \in M$. Then there exists a positive integer $r$ such that for $n>r A^{n} N \wedge N^{\prime}=A^{n-r}\left(A^{r} N \wedge N^{\prime}\right)$.

Proof. In [2] this result is proved for the case $L=M$.

Passing to $L \oplus M$, it follows that there exists an $r$ such that for $n>r$,

$$
\left((A, A \mathfrak{m})^{n}(O, N)\right) \wedge\left(O, N^{\prime}\right)=(A, A \mathfrak{m})^{n-r}\left((A, A \mathfrak{m})^{r}(O, N) \wedge\left(O, N^{\prime}\right)\right) .
$$

Simplifying both sides yields $\left(O, A^{n} N \wedge N^{\prime}\right)=\left(O, A^{n-r}\left(A^{r} N \wedge N^{\prime}\right)\right)$.

Thus for $n>r, A^{n} N \wedge N^{\prime}=A^{n-r}\left(A^{r} N \wedge N^{\prime}\right)$, q.e.d.

§ 3. Form lattices and localization. In this section two of the standard constructions of ring theory, form modules and localization, are abstracted to ring theoretic lattice modules. Throughout this section $L$ will be a Noether lattice and $M$ a Noetherian ring theoretic $L$-module. The convention $A^{i}=I$, for $i \leqslant 0$, will also be adopted.

Let $A \neq I \in L$. Then the form lattice of $L$ with respect to $A$, denoted $F(A)$, is the set of all formal sums $\sum_{i=0}^{\infty} B_{i}$, where $A^{i} \geqslant B_{i} \geqslant B_{i+1}$ $\geqslant A B_{i}$ and $B_{i} \geqslant A^{i+1}$, together with the following operations:

$$
\begin{gathered}
\left(\sum_{i} B_{i}\right) \vee\left(\sum_{i} C_{i}\right)=\sum_{i}\left(B_{i} \vee C_{i}\right), \\
\left(\sum_{i} B_{i}\right) \wedge\left(\sum_{i} C_{i}\right)=\sum_{i}\left(B_{i} \wedge C_{i}\right), \\
\left(\sum_{i} B_{i}\right)\left(\sum_{i} C_{i}\right)=\sum_{i} \bigvee_{k+j=i}\left(B_{k} C_{j} \vee A^{i+1}\right), \quad \text { and } \\
\sum_{i} B_{i} \leqslant \sum_{i} C_{i} \quad \text { if and only if } B_{i} \leqslant C_{i}, \quad \text { for all } i
\end{gathered}
$$


The form lattice of $M$ with respect to $A$, denoted $G(A, M)$, is the set of all formal sums $\sum_{i=0}^{\infty} N_{i}$, where $A^{i} \mathrm{~m} \geqslant N_{i} \geqslant N_{i+1} \geqslant A N_{i}$ and $N_{i} \geqslant A^{i+1} \mathrm{~m}$, together with the operations

$$
\begin{gathered}
\left(\sum_{i} N_{i}\right) \vee\left(\sum_{i} N_{i}^{\prime}\right)=\sum_{i}\left(N_{i} \vee N_{i}^{\prime}\right), \\
\left(\sum_{i} N_{i}\right) \wedge\left(\sum_{i} N_{i}^{\prime}\right)=\sum_{i}\left(N_{i} \wedge N_{i}^{\prime}\right), \quad \text { and } \\
\sum_{i} N_{i} \leqslant \sum_{i} N_{i}^{\prime} \quad \text { if and only if } N_{i} \leqslant N_{i}^{\prime}, \quad \text { for all } i .
\end{gathered}
$$

If $n$ is principal in $M$ and $n \leqslant A^{k} \mathrm{~m}, n \nless A^{k+1} \mathrm{~m}$, then the leading form of $n$, denoted $n^{\prime}$, is $\sum_{i=0}^{\infty}\left(A^{i-k} n \vee A^{i+1} \mathrm{~m}\right)$.

As might be supposed, the following is true.

THEOREM 3. $F(A)$ is a Noether lattice and $G(A, M)$ is a Noetherian ring theoretic $F(A)$-module under multiplication given by

$$
\left(\sum_{i} B_{i}\right)\left(\sum_{i} N_{i}\right)=\sum_{i} \underset{k+j=i}{\bigvee} B_{k} N_{j} \vee A^{i+1} \mathrm{~m} .
$$

Furthermore if $L$ is local, then $F(A)$ is local.

Proof. The easiest way to obtain this result is to apply the theory $A$-transforms for Noether lattices as developed by E. W. Johnson in [2]. Let $\Re(L, A)$ denote the $A$-transform of $L$ which Johnson proves to be a Noether lattice which is local when $L$ is local. In $\Re(L, A)$ consider the element $I^{(-1)}=\sum_{-\infty}^{\infty} B_{i}$, where $B_{i}=A^{i+1}$. Upon examination it is obvious that $\mathfrak{R}(L, A) / I^{(-1)}$ and $F(A)$ are isomorphic as multiplicative lattices under the correspondence $\sum_{i=-1}^{-\infty} I+\sum_{i=0}^{\infty} C_{i} \Leftrightarrow \sum_{i=0}^{\infty} C_{i}$. Thus $F(A)$ is a Noether
lattice since $\Re(L, A) / I^{(-1)}$ is a Noether lattice.

To get the module structure of $G(A, M)$ consider the Noether lattice $L^{\prime}=L \oplus M$ and then consider the form lattice $F^{\prime}\left(A^{\prime}\right)=F^{\prime}$ of $L^{\prime}$ where $A^{\prime}=(A, A \mathfrak{m})$. Now the interval $\sum_{i=0}^{\infty}\left(A^{i+1}, A^{i} \mathfrak{m}\right) / \sum_{i=0}^{\infty}\left(A^{i+1}, A^{i+1} \mathfrak{m}\right)$ is a ring theoretic $F^{\prime} / \sum_{i=0}^{\infty}\left(A^{i+1}, A^{i} \mathrm{~m}\right)$-module by Theorem 1 . Under the obvious correspondences $F^{\prime} / \sum_{i=0}^{\infty}\left(A^{i+1}, A^{i} \mathrm{~m}\right)$ is isomorphic to $F(A)$ and $\sum_{i=0}^{\infty}\left(A^{i+1}, A^{i} \mathfrak{m}\right) / \sum_{i=0}^{\infty}\left(A^{i+1}, A^{i+1} \mathfrak{m}\right)$ is isomorphic to $G(A, M)$. This completes the proof, q.e.d.

It should be pointed out that the previous theorem could also be proved by long and tedious computation. Indeed, it can be verified that leading forms of principal elements in $L$ and $M$ are principal in $F(A)$ and $G(A, M)$. Also straightforward computation shows that the leading forms of $M$-principal elements are $G$-principal. Furthermore an application of the Artin-Rees lemma (Corollary 2 above) can be made to show $F$ and $G$ Noetherian.

The basic idea for abstracting localization was given by Dilworth in [1]. Here Dilworth's results will be extended via the principle of idealization. The first step in the development of localization for $M$ is to note that the standard primary decomposition theorems for Noetherian modules over Noetherian commutative rings with identity also hold for $M$. The details of proof for this can be lifted easily from the standard proofs in ring theory (see for example [6]). Also this could be shown by using the principle of idealization and the well known results about primary decomposition in Noether lattices. For the remainder of this paper it will be assumed that the reader is conversant with this decomposition theory as well as the usual terminology of $P$-primary, $P$-component, and so forth.

Now let $D \in L$ and $N, N^{\prime}(\neq \mathrm{m}) \in M$ and define $N \sim N^{\prime}(D)$, if $N_{D}=N_{D}^{\prime}$, where $N_{D}$ denotes the isolated component of $N$ with respect to the sets of primes less than or equal to $D$; also set $\mathrm{m}_{D}=\mathrm{m}$. Also let $[N]$ denote the equivalence class of $N$ and let $M_{D}$ be the set of such equivalence classes. The following result describes the relation between $L_{D}$ and $M_{D}$.

THEOREM 4. $M_{D}$ is a Noetherian ring theoretic $L_{D}$-module under the operations

$$
\begin{aligned}
{\left[N_{1}\right] \vee\left[N_{2}\right] } & =\left[N_{1} \vee N_{2}\right], \\
{\left[N_{1}\right] \wedge\left[N_{2}\right] } & =\left[N_{1} \wedge N_{2}\right], \\
{[A][N] } & =[\dot{A} N] .
\end{aligned}
$$

Proof. The basic idea is to show that $\sim$ is a congruence relation with respect to join, meet, product, and residuation. The result then is immediate because then the above operations are well defined and satisfy the required properties of a ring theoretic $L_{D}$-module. In particular, equivalence classes containing principal ( $M$-principal) elements are principal ( $M$-principal) since principal ( $M$-principal) elements are defined by identities. 
The congruence properties of $\sim$ will be shown by passing to $L \oplus M$ and using the fact that $\sim$ is a congruence relation in a Noether lattice (this is shown in [1, p. 489]). The following is true and will be proved in a lemma below: $N \sim N^{\prime}(D)$ and $A \sim A^{\prime}(D)$ if and only $(A, N)$ $\sim\left(A^{\prime}, N^{\prime}\right)((D, \mathfrak{m}))$. Now assume $N_{1} \sim N_{1}^{\prime}, N_{2} \sim N_{2}^{\prime}$, and $A \sim A^{\prime}$. The congruence with respect to $\vee$ is shown in the following way: $\left(O, N_{1} \vee N_{2}\right)$ $=\left(O, N_{1}\right) \vee\left(O, N_{2}\right) \sim\left(O, N_{1}^{\prime}\right) \vee\left(O, N_{2}^{\prime}\right)=\left(O, N_{1}^{\prime} \vee N_{2}^{\prime}\right)$ by the above remark and the congruence property of $\sim$ in $L \oplus M$. Also by the above remark it follows that $N_{1} \vee N_{2} \sim N_{1}^{\prime} \vee N_{2}^{\prime}$. Similarly $N_{1} \wedge N_{2} \sim N_{1}^{\prime} \wedge N_{2}^{\prime}$. By the congruence of $\sim$ with respect to product in a Noether lattice $\left(O, A N_{1}\right)$ $=(A, \mathrm{~m})\left(0, N_{1}\right) \sim\left(A^{\prime}, \mathrm{m}\right)\left(O, N_{1}^{\prime}\right)=\left(O, A N_{1}^{\prime}\right)$. Thus $A N_{1} \sim A N_{1}^{\prime}$. It can also be shown similarly that $N_{1}: N_{2} \sim N_{1}^{\prime}: N_{2}^{\prime}$ and $N_{1}: A \sim N_{1}^{\prime}: A^{\prime}$. Hence $\sim$ satisfies the desired congruence properties, q.e.d.

Lemma. $(A, N) \sim\left(B, N^{\prime}\right)((D, \mathfrak{m}))$ if and only if $A \sim B(D)$ and $N \sim N^{\prime}(D)$

Proof. The following statements can be proved easily from the definitions of prime and primary elements. The prime elements of $L \oplus M$ are exactly those elements $(P, \mathfrak{m})$, where $P$ is prime in $L$. Furthermore primary elements in $L \oplus M$ are of the form $(N: \mathrm{m}, N)$ where $N$ is $P$-primary or $(Q, \mathrm{~m})$ where $Q$ is $P$-primary.

Now to prove the lemma it clearly suffices to show that $(A, N)_{(D, \mathrm{~m})}$ $=\left(A_{D}, N_{D}\right)$. Let $N=\bigwedge_{i=1}^{k} N_{i}$ and $A=\bigwedge_{i=k+1}^{n} Q_{i}$ be normal decompositions for $N$ and $A$, where $N_{i}$ is $P_{i}$-primary and $Q_{j}$ is $P_{j}$-primary. Now $(A, N)=\bigwedge_{j}\left(Q_{j}, \mathrm{~m}\right) \wedge \bigwedge_{i}\left(N_{i}: \mathrm{m}, N_{i}\right)$ since $A \mathrm{~m} \leqslant N$ implies that $N_{i}: \mathrm{m} \geqslant A$, for $i=1, \ldots, k$. By the remarks in the previous paragraph it follows that $\bigwedge_{j}\left(Q_{j}, \mathrm{~m}\right)$ is a normal decomposition for $(A, \mathrm{~m})$ and that $\bigwedge_{i}\left(N_{i}: \mathrm{m}, N_{i}\right)$ is a normal decomposition for $(N: \mathrm{m}, N)$. Now it can be easily proved from Lemma 5.2 of [2] that $(E \wedge F)_{D}=E_{D} \wedge F_{D}$, where $E, F$; and $D$ belong to an arbitrary Noether lattice. In particular, $(A, N)_{((D, \mathrm{mll})}=\bigwedge_{j}\left(Q_{j}, \mathfrak{m}\right)_{(D, \mathrm{ml})}$ $\wedge \bigwedge_{i}\left(N_{i}: \mathfrak{m}, N_{i}\right)_{(D, \mathrm{~m}))}=\left(A_{D} \wedge(N: \mathrm{m})_{D}, \mathfrak{m} \wedge N_{D}\right)=\left((A \wedge N: \mathfrak{m})_{D}, N_{D}\right)$. The last element equals $\left(A_{D}, N_{D}\right)$ because $A \leqslant N: \mathrm{m}$, q.e.d.

\section{References} [1] R. P. Dilworth, Abstract commutative ideal theory, Pacific J. Math. 12 (1962),
pp. 481-498.

[2] E. W. Johnson, A-Transforms and Hilbert functions in local lattices, to appear in Trans. Amer. Math. Soc.
[3] E. W. Johnson, Completions of Noetherian lattices, to appear.

[4] Masayoshi Nagata, Local Rings, New York 1961.

[5] D. G. Whitman, Ring Theoretic Lattice Modules and the Hilbert Polynomial, Thesis, University of California, Riverside 1968.

[6] O. Zariski and P. Samuel, Commutative Algebra, Vols. I and II, New York 1958.

\section{UNIVERSITY OF CALIFORNIA AT RIVERSIDE}

Riverside, California

Reģu par la Rédaction le 27. 3. 1969 